Applied Remote Sensing

\title{
Spatio-temporal features of vegetation restoration and variation after the Wenchuan earthquake with satellite images
}

Hou Peng

Wang Qiao

Yang Yipeng

Jiang Weiguo

Yang Bingfeng

Chen Qiang

Yuan Lihua

Kong Fanming

Chen Xi

Wang Guanjie 


\title{
Spatio-temporal features of vegetation restoration and variation after the Wenchuan earthquake with satellite images
}

\author{
Hou Peng, ${ }^{a}$ Wang Qiao, ${ }^{\text {a,* }}$ Yang Yipeng, ${ }^{a}$ Jiang Weiguo, ${ }^{\text {b }}$ Yang Bingfeng, ${ }^{c}$ \\ Chen Qiang, ${ }^{\mathrm{b}}$ Yuan Lihua, ${ }^{\mathrm{b}}$ Kong Fanming, ${ }^{\mathrm{b}}$ Chen Xi, ${ }^{\mathrm{b}}$ and Wang Guanjie ${ }^{\mathrm{d}}$ \\ ${ }^{a}$ Satellite Environment Center, Ministry of Environmental Protection of the People's Republic of \\ China, Beijing 100094, China \\ ${ }^{b}$ Beijing Normal University, Academy of Disaster Reduction and Emergency Management, \\ Beijing 100875, China \\ ${ }^{\mathrm{c} T s i n g h u a}$ University, School of Architecture, Beijing 100084, China \\ ${ }^{\mathrm{d}}$ China University of Mining and Technology, College of Geoscience and Surveying \\ Engineering, Beijing 100083, China
}

\begin{abstract}
The Wenchuan earthquake was a deadly earthquake that occurred on May 12, 2008, in Sichuan province of China. With the help of classic statistic methods, including arithmetic mean, standard deviation and linear trend estimation, vegetation restoration was recognized by analyzing spatio-temporal features of normalized difference vegetation index (NDVI) before and after this earthquake. Results indicate: (1) spatial distribution of NDVI mean values remains similar from 1998 to 2011. Higher values are mainly found in north, whereas lower values are mainly distributed over southeast, which is in good correlation with elevation and landform. Vegetation damage is at different levels in different seismic intensity (SI) regions: the higher SI is, the worse vegetation damage is. (2) Over the whole region, standard deviation is bigger after earthquake than before. Both absolute and relative changes in ecosystem stability increase with increasing SI. In different counties, variation of ecosystem stability is more obvious after earthquake, increase of standard deviation is approximately 6.5 times. Relatively, vegetation regionalization is the smallest analysis unit. Consequently, changes resulting from earthquake are unobvious. (3) Linear trend estimation coefficient increases from 0.0079 before the earthquake to 0.0359 after the earthquake in this whole region. This indicates that the plant ecosystem is rapidly restored between 2009 and 2011. The biggest linear trend is for the hill region, indicating good plant restoration and increase after earthquake. Fluctuation of linear trend estimation coefficient in different counties is more obvious after earthquake. Vegetation restoration after earthquake is most obvious in the regions that suffered the greatest SI (SI10 and SI11). In contrast, fluctuation in linear trend estimation coefficient of annual NDVI mean value for different classes of vegetation is more obvious before earthquake. (C) The Authors. Published by SPIE under a Creative Commons Attribution 3.0 Unported License. Distribution or reproduction of this work in whole or in part requires full attribution of the original publication, including its DOI. [DOI: 10.1117/1.JRS.8.083651]
\end{abstract}

Keywords: spatio-temporal feature; vegetation restoration; satellite images; Wenchuan earthquake.

Paper 13304 received Sep. 2, 2013; revised manuscript received Mar. 1, 2014; accepted for publication Mar. 10, 2014; published online Apr. 3, 2014.

\section{Introduction}

Vegetation is one of the most important components in a terrestrial ecosystem. It is regarded as the hinge of the cycle of mass and energy fluxes of ecosystem, and drives the evolvement and development of an ecosystem. ${ }^{1}$ Distribution and growth of vegetation are determined by environmental factors, such as water, heat energy, and nutrient availability, and their changes are logged by plant. Among these, water and heat availability are the most important. They are indicated by the rainfall

*Address all correspondence to: Wang Qiao, E-mail: wangqiao@sepa.gov.cn 
and air temperature, respectively. Because they are obvious or unobvious in different years, their correlation with vegetation growth paid the most attention over a long period. Popular study topics include the relationship between precipitation and the composition and distribution of vegetation population on an annual scale, spatio-temporal vegetation growth feature and rainfall and air temperature on season and month scale, ${ }^{2-5}$ and so on. Contrasted with natural hazards, annual variations of climate factors are gradual and gentle, simply influencing vegetation growth. However, many unexpected natural hazards, such as landslide, mudslide, earthquake, drought, flood and freezing, impact badly on the growth and spatial distribution of vegetation. ${ }^{6-9}$

Disaster not only affects ecological quality but also changes the ecosystem classifications. Usually, assessment of ecological damage induced by natural disasters focuses mainly on the measures of ecosystem conversion, that is to say, calculating the area reduced by the disaster. However, ecological restoration assessment is more important than ecological damage assessment. Vegetation is the most important ecological factor, and its damage assessment is very important. There is no doubt that timely monitoring of vegetation growth is the key to assess damage and evaluate vegetation restoration. Five and ten years after the 921 earthquake in central Taiwan, Lin et al. and Chuang et al. assessed the vegetation restoration with the Markov chain model and Systeme Probatoire d'Observation dela Tarre (SPOT) images. ${ }^{10,11}$ Although this case used model and satellite images, satellite images only record instantaneous vegetation information's, because that SPOT images are only acquired one time per month according to the devise of satellite. However, some satellite sensors, such as SPOT VEGETATION (VGT), moderate resolution imaging spectroradiometer (MODIS), images can be acquired two times per day according to the devise of satellite, 10-day satellite images can be gain and exactly reflect the real vegetation states. These satellite images were used to assess the vegetation restoration. ${ }^{12,13}$

After the earthquake occurred on May 12, 2008, in the Sichuan province of China, many researchers focused on this case. Xu et al. assessed the importance of ecological protection for ecological conservation, based on soil erosion sensitivity and the importance of biodiversity and water conservation. ${ }^{14}$ Kong et al. mainly analyzed the ecological vulnerability, based on resources and environment, and social and economic features. ${ }^{15} \mathrm{Xu}$ et al. mainly paid attention to the ecological influence in the epicenter region, with the help of remote sensing and geographical information systems. ${ }^{16}$ Wang et al. analyzed the correlation between geographical physiognomy and ecoenvironmental damage. ${ }^{17}$ Gao et al. used information extracted from satellite images taken on September 18, 2007, and July 28, 2008, to compare land use before and after earthquake. ${ }^{18}$ These studies mainly monitored and assessed ecological damage, and offered advice. However, 3 years after the earthquake, assessment of vegetation restoration after this natural disaster has been neglected, it is very important to monitor and assess changes in the ecosystem.

\section{Materials and Analytical Methods}

\subsection{Study Region}

The Wenchuan earthquake was measured $8.0 \mathrm{Ms}$ (surface wave magnitude scale). It occurred on May 12, 2008, in the Sichuan province of China. It is the most damaging and influential of all earthquakes that have occurred in China since 1949. Tremors were also felt in Vietnam, Thailand, Mongolia, Bangladesh, Nepal, India, Pakistan, and Russia. Ten counties that were worst hit were Wenchuan, Beichuan, Dujiangyan, Pengzhou, Shifang, Mao, An Xian, Pingwu, Qingchuan, and Mianzhu. ${ }^{19}$ A sketch of this epicenter and region is showed in Fig. 1. Mean altitude is $2034 \mathrm{~m}$ and landform is complex, with many hills in this region. According to the vegetation classification, there are about 18 kinds of plants. ${ }^{20}$ SI varied from SI7 to SI11, with the higher values occurring mainly in the east and south of this region. About $9.8 \%, 11.23 \%$, and $23.38 \%$ of this region experienced SI11, SI10, and SI9, respectively.

\subsection{Materials}

In order to clearly show vegetation restoration, normalized difference vegetation index (NDVI) images were collected from 1998 to 2011. A regional vegetation classification map, digital 
Peng et al.: Spatio-temporal features of vegetation restoration and variation after the Wenchuan earthquake...
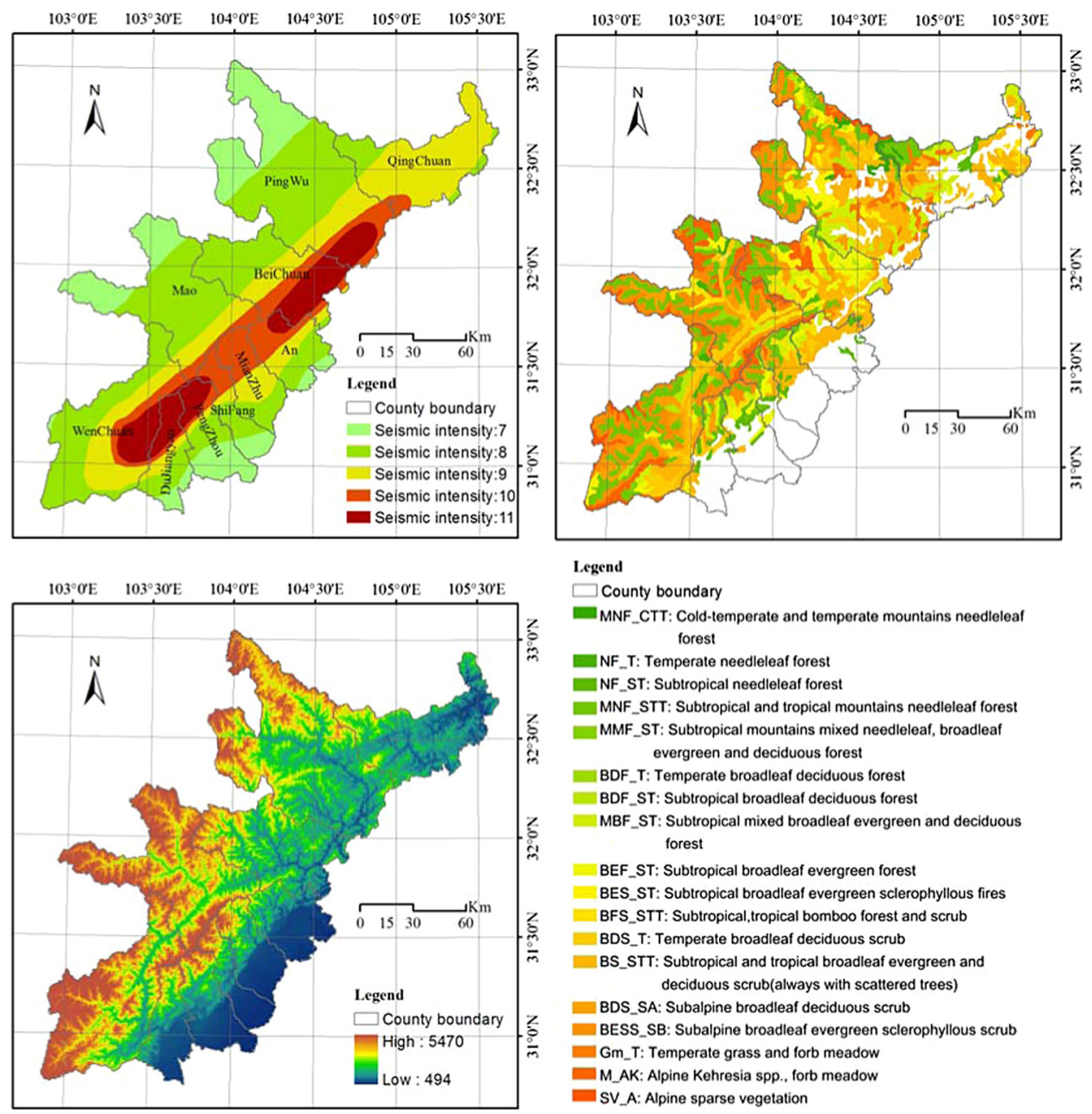

Fig. 1 Seismic intensity, geographical profile, and vegetation classification of the study region.

elevation model (DEM), and map of county boundaries were also collected. DEM data were downloaded from http://datamirror.csdb.cn/, they were acquired with shuttle radar topography mission (SRTM), produced by International Center for Tropical Agriculture (CIAT), and spatial resolution is $90 \mathrm{~m}$.

NDVI are satellite images with spatial resolution of $1 \mathrm{~km}$ acquired by SPOT VGT, they are the maximum-value composites synthesized from daily images, with the highest NDVI of an individual pixel for any given geographic coordinate, which helps to ensure coverage of all landmasses worldwide with a minimum cloud-cover effect. The platforms of SPOT VGT sensor are SPOT satellites (SPOT-4 and SPOT-5), which have an equator-crossing time of 10:30. The 10-day synthesis products until January 2003 are available at http://free.vgt.vito.be/origin from SPOT-4 (VGT1 sensor) and those after that from the SPOT-5 (VGT2 sensor). Real NDVI values are retrieved from original NDVI product images according to the equation $y=a x+b$, where $y$ is the NDVI value, $x$ is the digital number of the image, and $a$ and $b$ are 0.004 and -0.1 , respectively. ${ }^{21}$

\subsection{Methods}

Changes of many parameters were used to reflect vegetation restoration, such as growth, stability, and diversity. These can be recognized by the statistical features of long time series of NDVI images. Spatio-temporal features of vegetation before and after earthquake were analyzed by annual means of NDVI from 1998 to 2007 and from 2009 to 2011, respectively. Before calculating the statistic features of NDVI, average values of NDVI per year were made from 
10-day NDVI images over entire year. One year covers vegetation growth cycle in general, which can avoid errors caused by time differences of sprouting and deciduous of different vegetations. Based on these annual NDVI images, spatio-temporal features of vegetation restoration and variation were analyzed. After that, statistic images, including arithmetic mean, standard deviation and linear trend estimation of annual NDVI before and after earthquake, are analyzed with overlay methods based on the different counties, seismic intensity, and vegetation classification. By contrast, vegetation restoration and variation have been recognized and identified.

Arithmetic mean and standard deviation are the most commonly used statistical parameters. Arithmetic mean is the sum of a collection of numbers divided by the number of numbers in the collection, ${ }^{22}$ it is often used to report central tendencies. In this case, arithmetic mean was used to find the global central feature of annual NDVI in some regions or several years. Standard deviation is the square root of the variance of the random variable, statistical population, data set, or probability distribution. It shows how much variation or dispersion from the average exists. ${ }^{23}$ Standard deviation reflects individual dispersion in statistical samples, and thus indicates the statistical distribution and fluctuation amplitude in any region. According to the statistical signification, standard deviation was used to show the stability of vegetation.

It is not enough to analyze the vegetation restoration with arithmetic mean and standard deviation, because their values only showed the statistical distribution state, which does not indicate the global change in trend of variables with time. In order to recognize vegetation change and restoration state, linear trend estimation method was used to analyze the change trend. Linear trend estimation shows the change in trend of the temporal series of statistical variables. The linear trend estimation method analyzed the change in trend of interannual mean values using Eq. (1): ${ }^{23}$

$$
k=\frac{\sum_{i=1}^{n} x_{i} t_{i}-\frac{1}{n}\left(\sum_{i=1}^{n} x_{i}\right)\left(\sum_{i=1}^{n} t_{i}\right)}{\sum_{i=1}^{n} t_{i}^{2}-\frac{1}{n}\left(\sum_{i=1}^{n} t_{i}\right)^{2}},
$$

where $k$ is the linear trend estimation coefficient, which shows the change in trend of sample values, $x$ is the analysis sample value, $t$ is the time of sampling, and $n$ is the number of samples. Positive and negative $k$ is shown by the different signs. There is a rising trend when $k>0$, and there is a declining trend when $k<0$. The size of $k$ values shows the rate of rise or decline. The bigger the value of $k$ is, the larger the rate of change is.

\section{Results}

\subsection{General Features of Vegetation Variation}

According to statistic means of arithmetic mean, its value mainly reflects the center tendency of statistical variables, that is to say, mean value of annual NDVI over years. In detail, the bigger mean value of annual NDVI is, the better vegetation growth is. Figure 2 indicates a generally stable spatial distribution of mean NDVI values before and after earthquake. Higher values are mainly in the north, and lower values mainly distribute over the southeast, which is in good correlation with elevation and regional landform. In regions with higher elevation and complex landform, vegetation grows better. Specifically, the highest NDVI value is in Pingwu and the lowest value is in Shifang, corresponding to no difference in NDVI before and after the earthquake. Also, there is a minute change in the range of NDVI values. To be precise, the NDVI value varies from 0.0025 to 0.6666 before the earthquake, and from 0.0163 to 0.6807 after the earthquake. It is clear that both maximum and minimum are slightly increased by 0.0138 and 0.0141 , respectively. On the other hand, NDVI mean value over the whole region is decreased from 0.4553 to 0.4417 . The reduction is 0.0137 , about $3 \%$ of the NDVI mean value before the earthquake. Thus, there is an overall slight decrease in the general growth of vegetation in this region.

Regions differ in geological and geographical features, which result in spatial variation in the ability to withstand seismic activity. Therefore, it is reasonable to find that vegetation damage after earthquake is at different levels in different regions. In order to define this spatial variation, 
Peng et al.: Spatio-temporal features of vegetation restoration and variation after the Wenchuan earthquake...

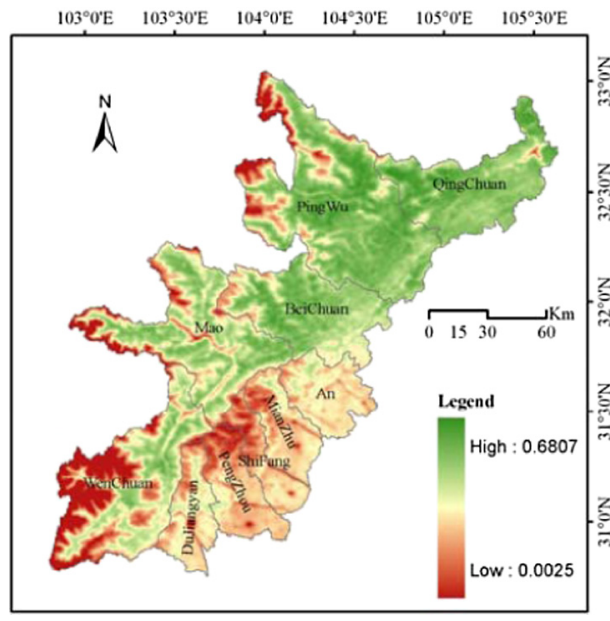

(a) Spatial distribution of NDVI mean value for 1998 to 2007 , before the earthquake

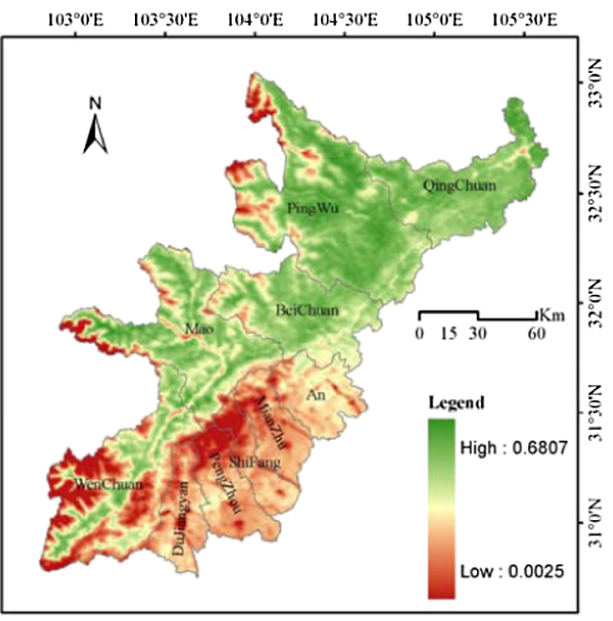

(b) Spatial distribution of NDVI mean value for 2009 to 2011, after the earthquake

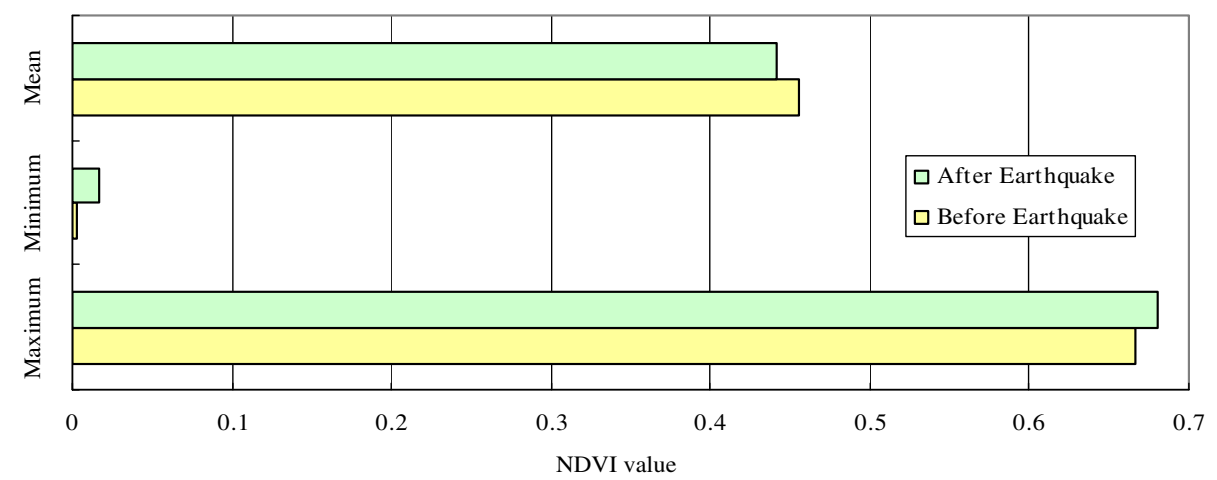

(c) Statistic feature of NDVI value before and after the earthquake

Fig. 2 NDVI mean value of vegetation restoration before and after the Wenchuan earthquake, (a) spatial distribution of NDVI mean value for 1998 to 2007, before the earthquake, (b) spatial distribution of NDVI mean value for 2009 to 2011, after the earthquake, and (c) static feature of NDVI value before and after the earthquake.

several types of maps are used to overlay the analysis: a map of political boundaries of counties, vegetation regionalization maps, and a seismic intensity distribution map. Figure 3 shows variation in mean values of NDVI before and after the earthquake (a) with seismic intensity, (b) by county, and (c) with vegetation type.

As well as the region where earthquake damage to vegetation occurs, the impact of seismic intensity on the vegetation growth must also be taken into account, and so the NDVI mean value is plotted against the variation in seismic intensity before and after the earthquake. In Fig. 3(a), it is seen that the higher the seismic intensity of the region, the worse the damage to vegetation. Typically, the greatest decrease in vegetation appears in regions of SI11 and SI10. It is about $9.8 \%$ in the SI11 region, which covers the earthquake center. According to the graph for the different counties [Fig. 3(b)], average NDVI has been in decline for many years, for example in An Xian, Mao Xian, Pingwu, Wenchuan, and more especially in Mianzhu, Shifang, Pengzhou, and Dujiangyan. The decline trend is more obvious in the northeast of this region, where the land is mainly cultivated. In general, a decline in different classes of vegetation is not observed after the earthquake, as shown in the plot of NDVI mean value against vegetation classification before and after the earthquake [Fig. 3(c)].

\subsection{Stability Variation in Different Regions}

The stability of a regional ecosystem is indicated by the stability of spatial distribution. Standard deviation is calculated with annual NDVI and used as the indication index: the bigger the 


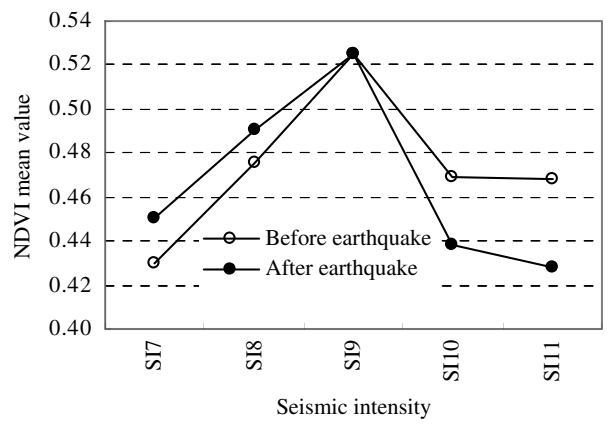

(a) Plot of NDVI mean value against seismic intensity

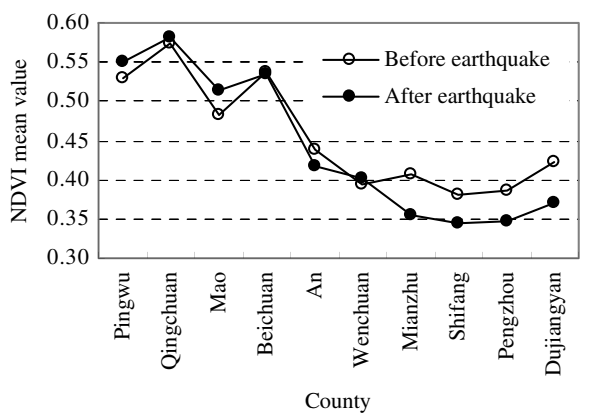

(b) Plot of NDVI mean value in different counties

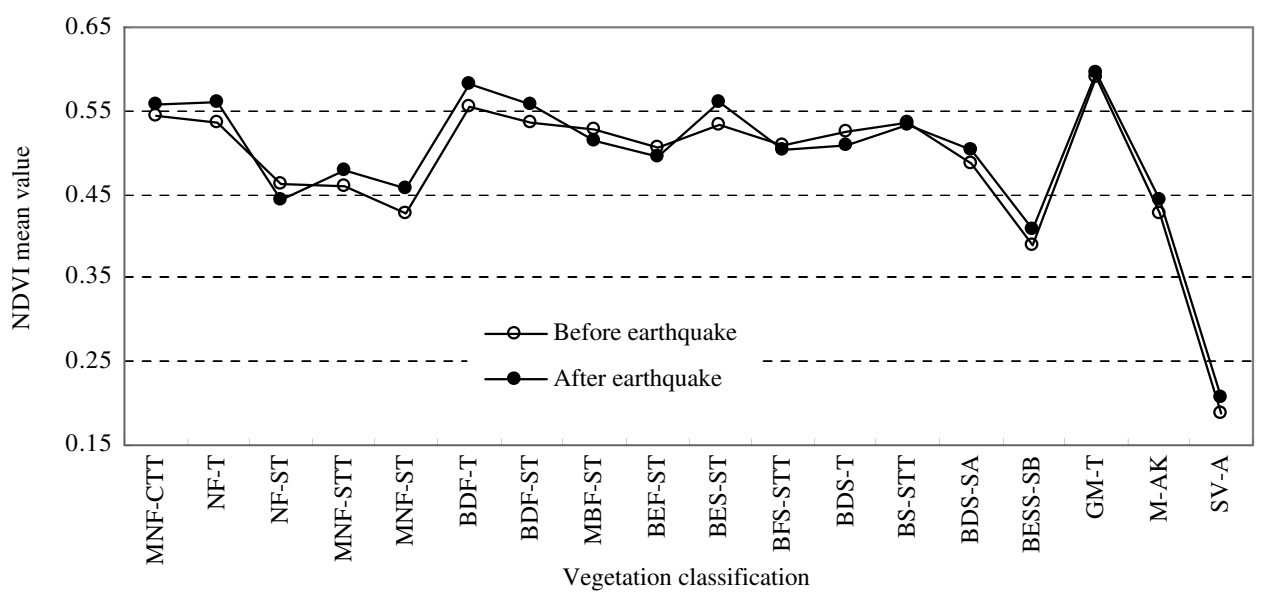

(c) Plot of NDVI mean value for different classes of vegetation

Fig. 3 Spatial variation of NDVI mean value of vegetation restoration before and after earthquake, (a) plot of NDVI mean value against seismic intensity, (b) plot of NDVI mean value in different counties, and (c) plot of NDVI mean value for different classes of vegetation.

standard deviation and the less stable the regional ecosystem. Adversely, the regional ecosystem is more stable. This index is clearly and closely linked to the area under assessment. Figure 4 shows the variation in standard deviation with (a) seismic intensity, (b) county, and (c) vegetation type. In general, the standard deviation is bigger after than before the earthquake. Both absolute and relative changes in stability increase with seismic intensity, being most obvious in the SI11 region [Fig. 4(a)].

As Fig. 4(b) indicates, differences in stability before and after the earthquake are evident in different counties. The biggest absolute changes are in Qingchuan and Beichuan from 0.0424 to 0.5815 and from 0.0599 to 0.5355 , respectively. The greatest relative changes appear in Qingchuan and Anxian, standard deviations after the earthquake being 12.7303 and 13.3132 times those before the earthquake, respectively. These values indicate that there are obvious changes in Qingchuan, Beichuan, and Anxian, the ecosystem being less stable after the earthquake. In general, ecosystem stabilities in different counties are similar before the earthquake; stability variation is greater after the earthquake. In different counties, variation of ecosystem stability is more obvious after the earthquake, increase of standard deviation is approximately 6.5 times. Overall, the ecosystem is less stable in the north than in the south. Absolute changes show the same spatial distribution.

Relatively, vegetation regionalization is the smallest analysis unit. As a result, differences between before and after the earthquake are not obvious. However, stability variation is the most important in understanding the ecosystem change in this earthquake region. As Fig. 3(c) indicates, there are obvious changes in MNF_CTT, NF_T, NF_ST, MNF_STT, MMF_ST, MBF_ST, BEF_ST, BFS_STT, BS_STT, BDS_SA, and GM_T. The greatest absolute changes appear in MNF_CTT and MMF_ST: 0.0235 and 0.0343 , respectively. The greatest relative changes are in MMF_ST and BFS_STT. In general, regions of obvious change are mainly found at higher altitudes, that is to say, in the west of the study region. 


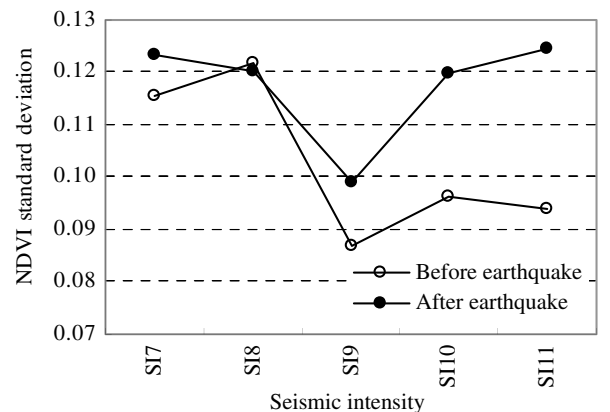

(a) Plot of NDVI standard deviation against seismic intensity

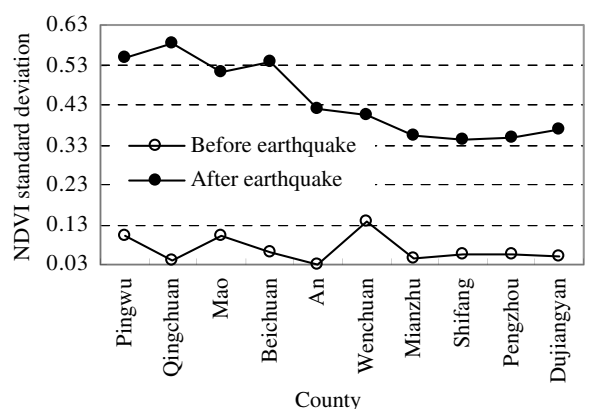

(b) Plot of NDVI standard deviation in different counties

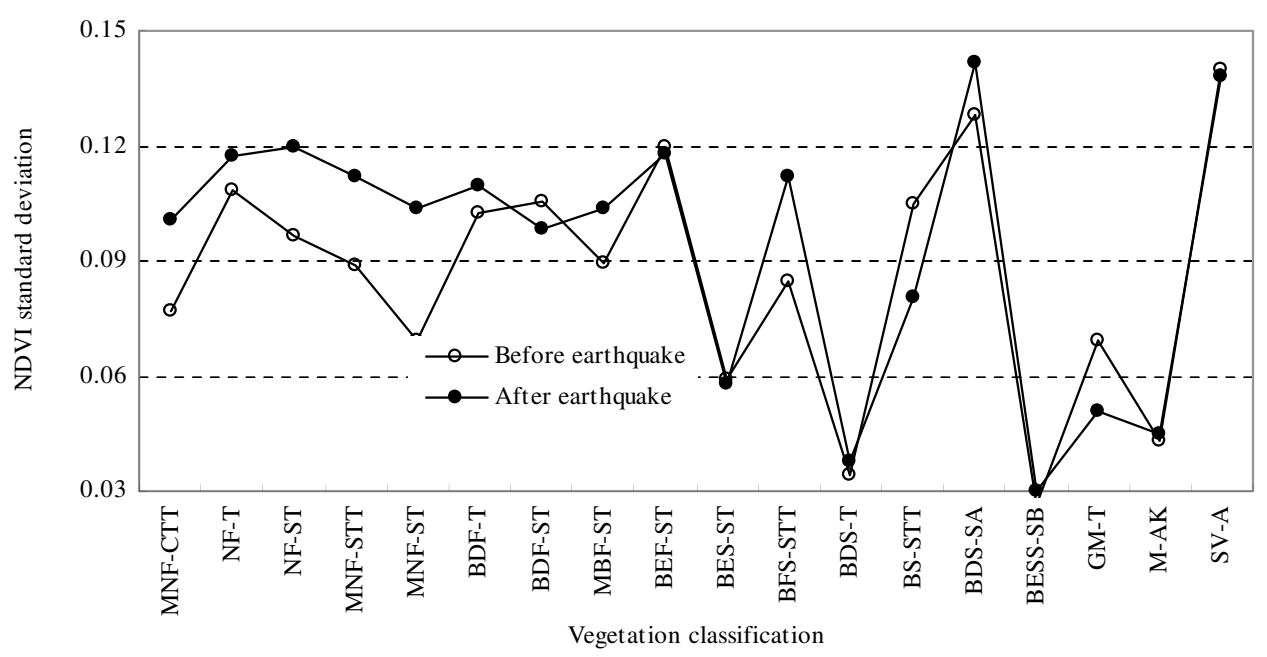

(c) Plot of NDVI standard deviation for different classes of vegetation

Fig. 4 Spatial variation of NDVI standard deviation of vegetation restoration before and after earthquake, (a) plot of NDVI standard deviation against seismic intensity, (b) plot of NDVI standard deviation in different counties, and (c) plot of NDVI standard deviation for different classes of vegetation.

\subsection{Variation in Restoration of Vegetation in Different Regions}

Although a vegetation ecosystem undoubtedly fluctuates between different years, this change is minute in a normal year. When a major natural disaster happens, the path of general change is from good to bad and then back to good. In this process, vegetation growth change is one of the most useful indices for monitoring and assessing the restoration of vegetation. In order to show the variation in restoration after the earthquake by area, the linear trend estimation coefficient is taken as the indicator. The variation of linear trend estimation coefficient over the different regions is shown in Figs. 5 and 6. Over the whole region, linear trend estimation coefficient increases from 0.0079 before the earthquake to 0.0359 after the earthquake. Such a large change indicates that the plant ecosystem is in the process of rapid restoration from 2009 to 2011. As with the spatial distribution before the earthquake, the biggest and smallest linear trend estimation coefficients are found in the eastern, cultivated, vegetation; and the western hill vegetation, which reflects the natural hill flora, is stable. However, the biggest linear trend estimation coefficient is mainly scattered over the hill region, indicating that plant restoration and increase after the earthquake are good in this region.

In order to clearly show the variation in restoration in different regions, graphs of linear trend estimation coefficient in different counties (a), with seismic intensity (b) and with vegetation type (c) are shown in Fig. 6. Bigger line trends and good plant restoration are shown in these figures; however, fluctuation in linear trend estimation coefficient in different counties is more obvious after the earthquake than before the earthquake. Except in Qingchuan, the vegetation shows a bigger linear trend estimation coefficient value and better growth in all counties. The changes are more obvious, especially in Wenchuan and Maoxian, than before the 
Peng et al.: Spatio-temporal features of vegetation restoration and variation after the Wenchuan earthquake...

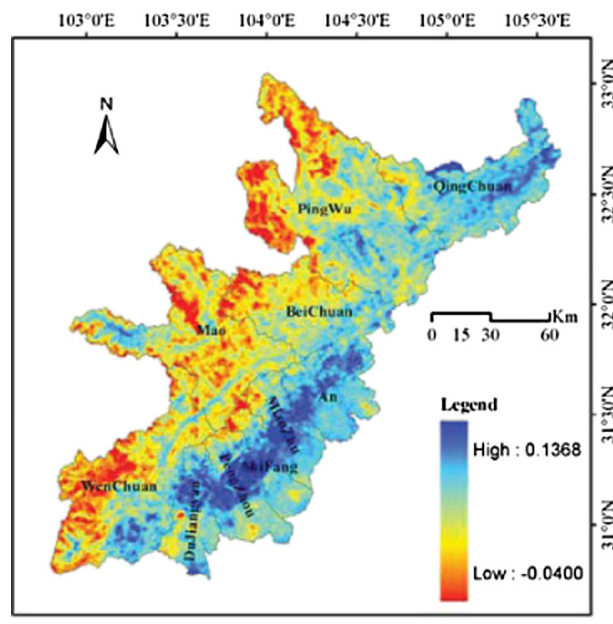

(a) Spatial distribution of linear trend estimation coefficient of annual NDVI mean value before the earthquake

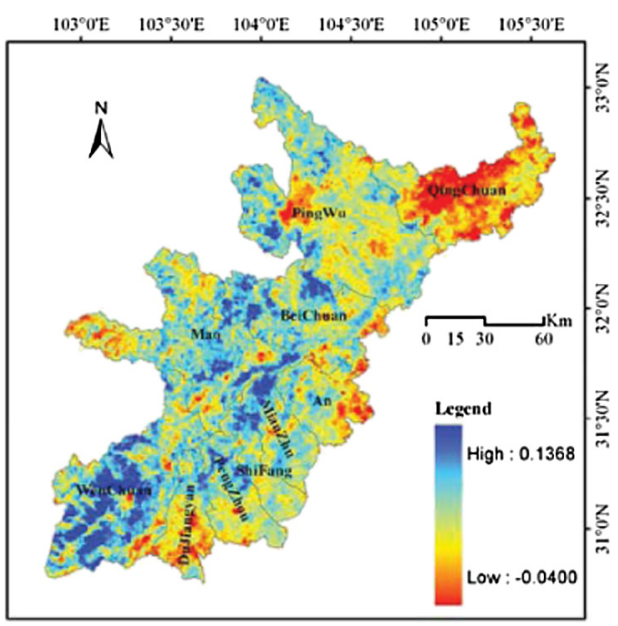

(b) Spatial distribution of linear trend estimation coefficient of annual NDVI mean value after the earthquake

Fig. 5 NDVI linear trend estimation coefficient of vegetation restoration before and after earthquake, (a) spatial distribution of linear trend coefficient of annual NDVI mean value before the earthquake and (b) spatial distribution of linear trend coefficient of annual NDVI mean value after the earthquake.

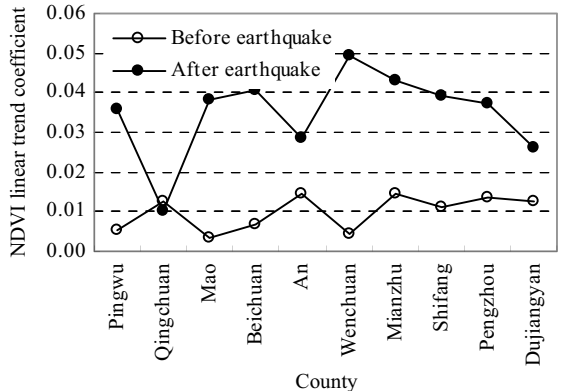

(a) Plot of linear trend estimation coefficient of annual NDVI mean value in different counties

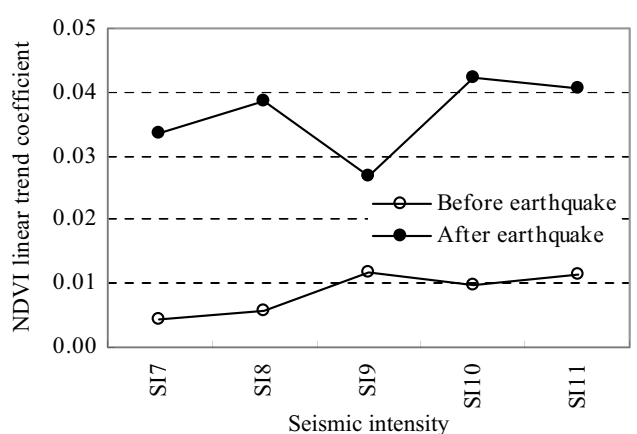

(b) Plot of linear trend estimation coefficient of annual NDVI mean value against seismic intensity

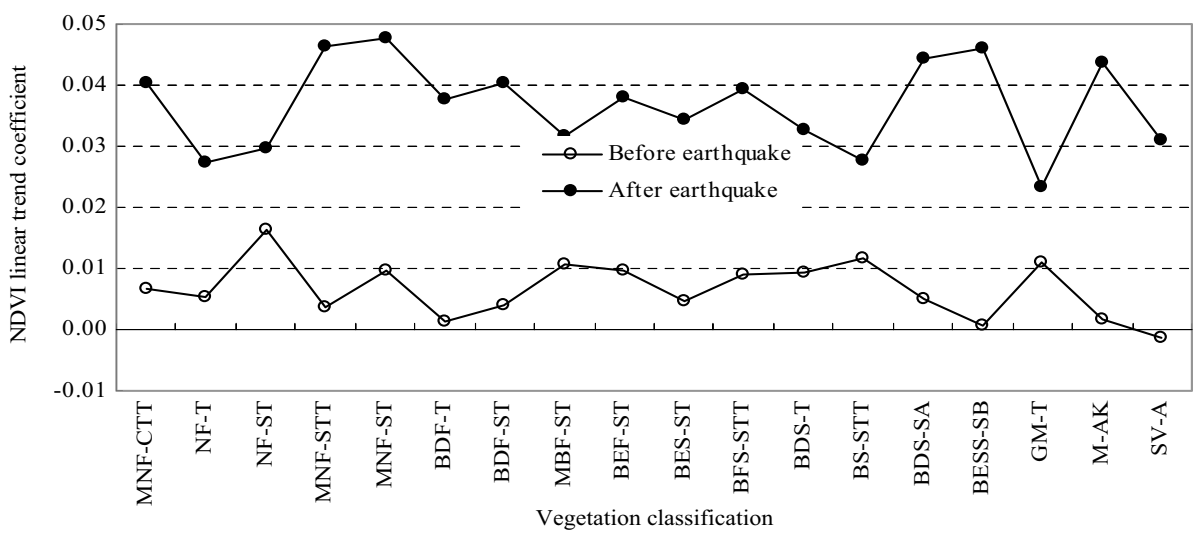

(c) Plot of linear trend estimation coefficient of annual NDVI mean value for different vegetation classes

Fig. 6 Linear trend estimation coefficient of vegetation restoration after earthquake, (a) plot of linear trend estimation coefficient of annual NDVI mean value in different counties, (b) plot of linear trend estimation coefficient of annual NDVI mean value against seismic intensity, and (c) plot of linear trend estimation coefficient of annual NDVI mean value for different vegetation classes. 
earthquake; the absolute increases are 0.0447 and 0.0350 , and the relative increases are 9.8887 and 10.5814, respectively. Among the regions of different seismic intensity, restoration of vegetation is the most obvious in SI10 and SI11 regions. However, the biggest absolute and relative increases occur in areas with SI8 and SI7: 0.0330 and 0.0292, respectively.

In contrast, the graphs of linear trend estimation coefficient of annual NDVI mean values for different classes of vegetation show greater fluctuation before the earthquake. The highest and lowest values are for different vegetation classifications. Vegetation growth is best for NF_ST before and MMF_ST after the earthquake, and worst for SV_A before and GM_T after the earthquake, the increase being $0.0132,0.0379,0.0323$, and 0.0123 , respectively, for these vegetation types. Over all the regions, the growth shows the greatest change for BESS_SB, i.e., 0.0453, 72.0954 times the growth before the earthquake. There is also a large increase in MNF_STT and SV_A. According to Fig. 6(c), vegetation types MNF_STT, MMF_ST, BDS_SA, BESS_SB, and M_AK are growing well.

\section{Conclusion and Discussions}

The Wenchuan earthquake was taken as the case study for this paper. This deadly earthquake, measuring 8.0 Ms, occurred on May 12, 2008, in the Sichuan province of China. Spatio-temporal features of vegetation restoration and variation after the Wenchuan earthquake were analyzed by arithmetic mean, standard deviation, and linear trend estimation of NDVI satellite images from 1998 to 2011. Main conclusions are concisely formulated as: vegetation damage is at different levels in different seismic intensity regions, the higher seismic intensity is, the worse vegetation damage is. Variation of ecosystem stability in different seismic intensity and counties is more obvious after earthquake. Plant ecosystem is rapidly restoring and increasing between 2009 and 2011 in the whole region, which is spatial variation, obvious restoration is in the hill region, greatest seismic intensity.

Before and after the earthquake, the spatial distribution of NDVI mean values is similar for many years. Higher values of NDVI are mainly found in the north, and lower values mainly in the southeast, which is in good correlation with the altitude and landform. On the other hand, different regions vary geologically and geographically, which result in spatial variation in the ability to withstand seismic activity in different regions, and so vegetation damage due to the earthquake is at different levels in different regions. In general, standard deviation is bigger after the earthquake than before. Both absolute and relative changes in stability increase with the seismic intensity, the most obvious changes occurring in the SI11 region. Ecosystem stability in different counties is similar before the earthquake, but variation in stability is evident after the earthquake. The ecosystem is less stable in the north than in the south. Absolute changes show the same spatial distribution. Relatively, vegetation regionalization is the smallest analysis unit. As a result, changes resulting from the earthquake are unobvious. Over the whole region, linear trend estimation coefficient increases from 0.0079 before the earthquake to 0.0359 after the earthquake. This large difference indicates that the plant ecosystem is in the process of rapid restoration between 2009 and 2011. The biggest linear trend estimation coefficient is mainly scattered over the hill region, indicating that plant restoration and increase after the earthquake are good in this region. Fluctuation of linear trend estimation coefficient between different counties is more obvious after the earthquake. Vegetation restoration is greatest in the regions that suffered the highest seismic intensities, SI10 and SI11. In contrast, the fluctuation in linear trend estimation coefficient of annual NDVI mean value for different classes of vegetation is more obvious before the earthquake.

Notwithstanding, some conclusions have been drawn from this study case, some further studies and analysis are needed to be done in the future. For example, to recognize the restoration difference of natural and crops is also very important for ecological restoration estimation after the earthquake. Because of the limit of resolution of satellite images and smaller cultivated land in the hills resulting in mixed pixels of natural vegetation and crops, it is almost impossible to recognize this difference in this paper. Moreover, to find which type vegetation is more appropriate for ecological reconstruction in this region maybe more important. Maybe this can be done with satellite images in higher spatial resolution. On the other hand, to find possible reasons for 
Peng et al.: Spatio-temporal features of vegetation restoration and variation after the Wenchuan earthquake...

the vegetation restoration and variation, that is to say, the driving force of vegetation variations is more important to direct the ecology reconstruction and restoration. For example, to find the relationships between the vegetation restoration and climatic (rainfall and air temperature), soil nutrient and chemical component are also very important. Of course, to find vegetation changes is the first step and premise.

\section{Acknowledgments}

Funds for this work are provided by Environmental nonprofit specific research project (2011467026).

\section{References}

1. H. Q. Jiang et al., Plant Ecology, 2nd ed., Higher Education Press, Beijing (2010).

2. T. L. Ackerman, "Germination and survival of perennial plant species in the Mojave Desert," The Southwest Nat. 24(3), 399-408 (1979), http://dx.doi.org/10.2307/3671296.

3. R. L. Specht and A. Specht, "Canopy structure in eucalyptus-dominated communities in Australia along climatic gradients," Acta Oecologica, Oecologia Plantarum 10(2), 191213 (1989).

4. J. M. K. Onema and A. Taigbenu, "NDVI-rainfall relationship in the Semliki watershed of the equatorial Nile," Phys Chem Earth, Parts A/B/C 34(13-16), 711-721 (2009), http://dx .doi.org/10.1016/j.pce.2009.06.004.

5. P. Hou et al., "Sensitivity analyses of different vegetations responding to climate change in inland river basin of China," J. Geogr. Sci. 22(3), 387-406 (2012), http://dx.doi.org/10 .1007/s11442-012-0934-1.

6. B. S. Caruso, C. Pithie, and L. Edmondson, "Invasive riparian vegetation response to flow regimes and flood pulses in a braided river floodplain," J. Environ. Manage. 125, 156-168 (2013), http://dx.doi.org/10.1016/j.jenvman.2013.03.054.

7. B. Crouzy et al., "Impact of floods on the statistical distribution of riverbed vegetation," Geomorphology 202, 51-58 (2013), http://dx.doi.org/10.1016/j.geomorph.2012.09.013.

8. H. Osvel et al., "Reprint of: effects of drought on birds and riparian vegetation in the Colorado River Delta, Mexico," Ecol. Eng. 59, 104-110 (2013), http://dx.doi.org/10 .1016/j.ecoleng.2013.01.001.

9. P. Cui, Y. Lin, and C. Chen, "Destruction of vegetation due to geo-hazards and its environmental impacts in the Wenchuan earthquake areas," Ecol. Eng. 44, 61-69 (2012), http:// dx.doi.org/10.1016/j.ecoleng.2012.03.012

10. C. Lin et al., "Vegetation recovery assessment at the Jou-Jou Mountain landslide area caused by the 921 earthquake in central Taiwan," Ecol. Model. 176(1-2), 75-81 (2004), http://dx.doi.org/10.1016/j.ecolmodel.2003.12.037.

11. C. Chuang et al., "Application of Markov-chain model for vegetation restoration assessment at landslide areas caused by a catastrophic earthquake in central Taiwan," Ecol. Model. 222(3), 835-845 (2011), http://dx.doi.org/10.1016/j.ecolmodel.2010.11.007.

12. Z. Sun et al., "Evaluation of ecological restoration through vegetation patterns in the lower Tarim River, China with MODIS NDVI data," Ecol. Informatics, 6(2), 156-163 (2011), http://dx.doi.org/10.1016/j.ecoinf.2010.10.002.

13. Z. Wu et al., "Increasing terrestrial vegetation activity of ecological restoration program in the Beijing-Tianjin Sand Source Region of China," Ecol. Eng. 52, 37-50 (2013), http://dx .doi.org/10.1016/j.ecoleng.2012.12.040.

14. W. Xu et al., "Assessnent of ecological protection in importance for ecological conservation in Wenchuan earthquake hard-hit disaster areas," Acta Ecologica Sinica. 28(12), 5820-5825 (2008).

15. B. Kong et al., "Appraisal of ecological vulnerability degree in Wenchuan earthquake area," Bull. Soil Water Conserv. 30(6), 180-184 (2010).

16. X. Xu et al., "Assement about the impact of Wenchuan earthquake on ecological environment," Acta Ecologica Sinica 28(12), 5989-5908 (2008). 
Peng et al.: Spatio-temporal features of vegetation restoration and variation after the Wenchuan earthquake...

17. Q. Wang et al., "The characters of relief amplitude in the severest disaster area of Wenchuan earthquake and its association analysis with the eco-environental damage," Sichuan Environment 28(5), 44-49 (2009).

18. H. Gao et al., "The post-earthquake landscape pattern changes of land use in northern mountain areas of Mianzhu," Remote Sens. Land Resour. 22(2), 97-101 (2010), http://dx.doi.org/ 10.6046/gtzyyg.2010.02.21.

19. "Information Column of 5.12 WenChuan Earthquake," http://www.cea.gov.cn/manage/ html/8a8587881632fa5c0116674a018300cf/sc/index.html (2008).

20. Editorial committee of vegetation map of China, Chinese Academy of Sciences, Vegetation Map of the People's Republic China (1:1000000), Chinese Geological Publishing House, Beijing (2007).

21. Institute for Remote Sensing Applications VEGETATION International Users Committee, Secretariat, "VEGETATION" Onboard SPOT 4 Product Specifications, 2nd ed., Ispra (1994).

22. H. R. Jacobs, Mathematics: A Human Endeavor, 3rd ed., W. H. Freeman, New York (1994).

23. F. Y. Wei, Modern Climatological Statistical Diagnosis and Prediction Methods, 2nd ed., China Meteorological Press, Beijing (2007).

Hou Peng is senior engineer in the National Environmental Satellite Remote Sensing Laboratory at the Satellite Environment Center, Ministry of Environmental Protection, the People's Republic of China. He is mainly engaged in the application of environmental and ecological remote sensing, ecosystem management, and assessment of ecosystem services.

Wang Qiao is a professor in the National Environmental Satellite Remote Sensing Laboratory at the Satellite Environment Center, Ministry of Environmental Protection, the People's Republic of China. He is mainly engaged in the principle research and application of environmental remote sensing, environmental monitoring, and environmental information.

Yang Yipeng is a professor in the National Environmental Satellite Remote Sensing Laboratory at the Satellite Environment Center, Ministry of Environmental Protection, the People's Republic of China. He is mainly engaged in the application of environmental remote sensing, and principle research and application of unpiloted airplane remote sensing.

Biographies of the other authors are not available. 